\title{
The Strange Story of BI Lyncis: an Extremely Helium-Rich Subdwarf with a Cool Companion
}

\author{
C. Simon Jeffery, R. Aznar Cuadrado \\ Armagh Observatory, College Hill, Armagh BT61 9DG, Northern \\ Ireland
}

\begin{abstract}
The star BI Lyn $=$ PG $0900+400$ was previously classified as an evolved binary system containing either a hot subdwarf or white dwarf and a thick accretion disk. We have analyzed new intermediate dispersion spectra and demonstrated that both views are wrong. BI Lyn is a highly-evolved system containing a luminous low-mass helium star and a rapidly rotating G-type giant. It may be closely related to the evolved sdO+G binary HD 128220 .
\end{abstract}

\section{Introduction}

The most unusual stars are frequently to be found in binary systems and to have properties that are, superficially at least, unique. Sometimes this may be a result of having too much data, sometimes too little. Another consequence of having too little data is that an understandable misinterpretation of low-quality data can grow into a full-blown myth in which a star takes on a persona quite divorced from its true nature. In a recent paper (Jeffery \& Aznar Cuadrado 2001), one such impostor, BI Lyncis - allegedly a nova-like cataclysmic variable, was unmasked. This poster paper summarises the main points of its dénouement as a low-mass supergiant binary with an extremely evolved hot component.

\section{The story so far}

The star PG 0900+400 was identified in the Palomar-Green survey as a subdwarf B (sdB) star with an infrared excess (Ferguson, Green, \& Liebert 1984), although the overall flux distribution was notably redder than seen in other composite systems. As such it was proposed to be a potential cataclysmic variable (CV) progenitor. Photometric monitoring subsequently identified a $0.34 \mathrm{~d}$ period (Lipunova \& Shugarov 1990, 1991; Kuczawska, Mikolajewski, \& Kirejczyk 1993), from which the designation nova-like CV seems to have arisen (Downes, Webbink, \& Shara 1997). This assignment may have been compounded by the presence of a $\mathrm{P}$ Cygni type line profile at $\mathrm{H} \alpha$, with a variable absorption component (Wade \& Potter 1995). Most recently, it was suspected to be a velocity variable (Orosz, Wade, \& Harlow 1997). Successive spectral decompositions indicated that the components were a subdwarf B star and a K3 dwarf (Ferguson et al. 1984; Orosz et al. 1997; Liu \& Hu 2000), perhaps because several similar 


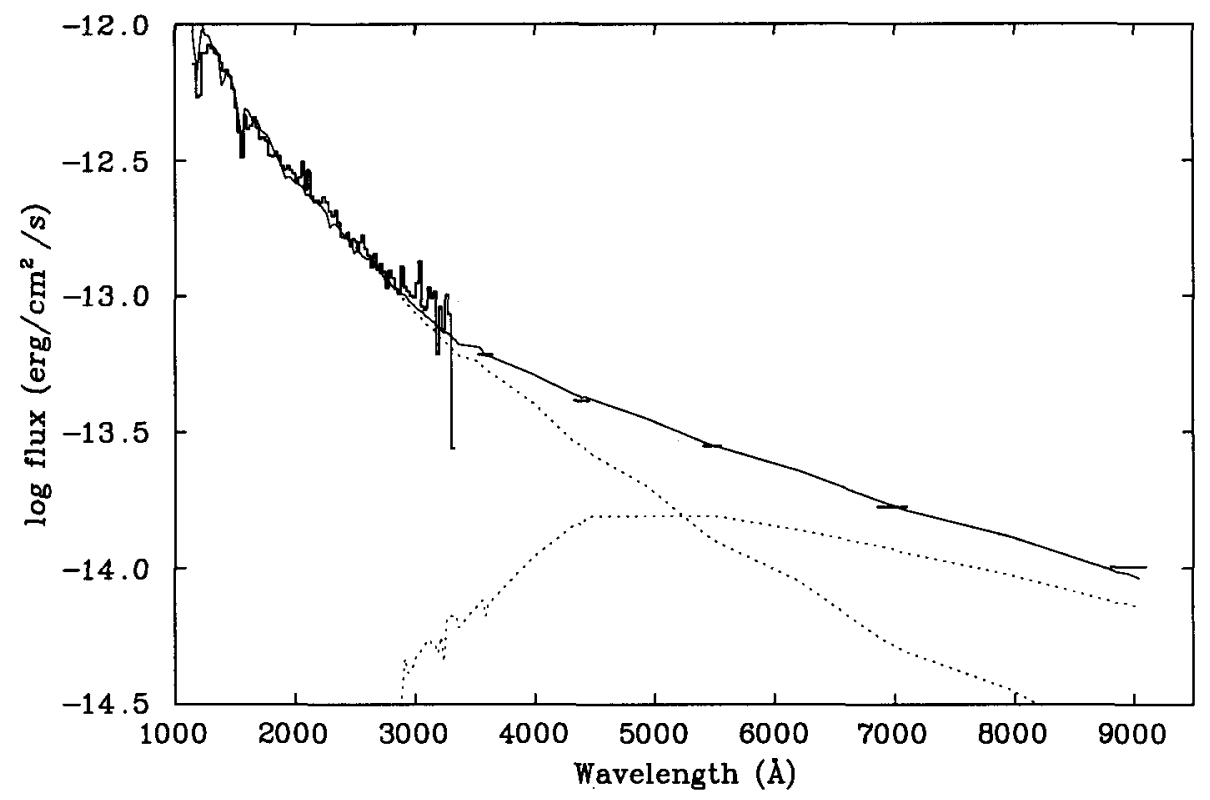

Figure 1. Ultraviolet and visual spectrophotometry of BI Lyn (histogram) together with the best fitting theoretical flux distribution (polyline and horizontal bars). The latter represents the sum of two model atmospheres (dashed lines) with $T_{\text {eff: } 1}=28600 \mathrm{~K}, \theta_{1}=$ $0.55 \times 10^{-11} \mathrm{rad}, T_{\text {eff: } 2}=5840 \mathrm{~K}$ and $\theta_{2}=4.09 \times 10^{-11} \mathrm{rad}$. Interstellar reddening is negligible.

such systems were well known. However reports of strong HeI lines (Orosz et al. 1997, Liu \& Hu 2000) had not triggered any particular alarm bells.

Our own encounter with this star was almost accidental. We had undertaken a spectroscopic survey of sdB stars with composite spectra (Aznar Cuadrado \& Jeffery 2002). PG $0900+400$ featured as a target but, in preparing the survey sample, we had omitted a complete literature survey for all potential targets. Had we encountered the SIMBAD designation " $V *$ BI Lyn - Nova-like Star," our target selection might have been different.

\section{Observations}

Moderate-resolution blue spectra of a large number of sdB stars were obtained with the Isaac Newton Telescope of the La Palma Observatory. The spectrum of PG $0900+400$ was interesting because, instead of showing broad Balmer lines and not much else as anticipated, it had a very line-rich spectrum. Initially it was thought to be the spectrum of a $\mathrm{G}$ star and possibly a wrong target identification. Closer inspection revealed that the strongest lines were due to He I, with weaker and quite narrow Balmer lines. It was more recognizable as an extreme helium star than as a subdwarf B star. The spectrum also contained evidence of a 
G-band, calcium $\mathrm{H}+\mathrm{K}$ lines and the near infrared calcium triplet - concrete evidence for the presence of a late-type companion (Jeffery \& Pollacco 1988). Subsequently, higher-resolution spectra were obtained with the William Herschel Telescope in order to measure the atmospheric parameters more accurately. To supplement the spectroscopic data, published photometry (Ferguson et al. 1984; Lipinova \& Shugarov 1991) and IUE images provided a description of the overall flux distribution from $1100-9000 \AA$.

Table 1. Atmospheric parameters for BI Lyn.

\begin{tabular}{|c|c|c|c|c|c|}
\hline Star & 1 & \pm & 2 & \pm & \\
\hline \multicolumn{6}{|c|}{ Spectrophotometry (FFIT) } \\
\hline$E_{\mathrm{B}-\mathrm{V}}$ & 0.00 & 0.02 & & & \\
\hline$T_{\text {eff }}$ & 28.6 & 1.0 & 5.84 & 0.96 & \\
\hline$\theta$ & 0.55 & 0.01 & 4.09 & 0.10 & $10^{-11} \mathrm{rad}$ \\
\hline$v_{t}$ & 5 & $a$ & 2 & $a$ & $\mathrm{~km} \mathrm{~s}^{-1}$ \\
\hline$R / R_{1}$ & 1 & & 7.44 & 0.03 & \\
\hline \multicolumn{6}{|c|}{ Spectroscopy (SFIT) } \\
\hline$\left(T_{\text {eff }}\right.$ & 30.1 & 0.01 & & & $\mathrm{kK})^{b}$ \\
\hline$T_{\text {eff }}$ & 28.6 & $a$ & 5.84 & $a$ & $\mathrm{kK}$ \\
\hline $\log g$ & 3.6 & 0.1 & 3.2 & 0.3 & (cgs) \\
\hline$n_{\mathrm{He}}$ & 0.95 & 0.01 & 0.1 & $a$ & \\
\hline$\left[\beta_{\mathrm{Fe}}\right]$ & 0.0 & $a$ & 0.0 & $a$ & \\
\hline$v \sin i$ & 0 & $a$ & 120 & 20 & $\mathrm{~km} \mathrm{~s}^{-1}$ \\
\hline & 5 & $a$ & 2 & $a$ & $\mathrm{kms}^{-1}$ \\
\hline$R / R_{1}$ & 1 & & 4.9 & 0.5 & \\
\hline
\end{tabular}

\section{Atmospheric parameters}

We first carried out a reanalysis of the UV and optical flux distribution to measure the effective temperatures $\left(T_{\text {eff }}\right)$ and angular diameters $(\theta)$ of both stars (Fig. 1). For this we used the automatic flux fitting package FFIT and a combination of hydrogen-deficient model atmospheres for hot stars and Kurucz model atmospheres for cool stars (cf. Aznar Cuadrado \& Jeffery 2001).

The new INT and WHT intermediate resolution spectra of BI Lyn in the blue (Fig. 2) and around $\mathrm{H} \alpha$ and the infrared calcium triplet were subsequently used to carry out a simultaneous spectral analysis of both stars using the automatic fitting package SFIT. Parameters to be solved for included $T_{\text {eff }}$ (although only that obtained from spectrophotometry was ultimately used), surface gravity $(\log g)$, relative abundance of helium by number $\left(n_{\mathrm{He}}\right)$, logarithmic mass fraction of metals relative to solar $\left(\left[\beta_{\mathrm{Fe}}\right]\right)$, rotational velocity, $(v \sin i)$ and relative radii $\left(R / R_{1}\right)$. The results of both analyses are given in Table 1 . 

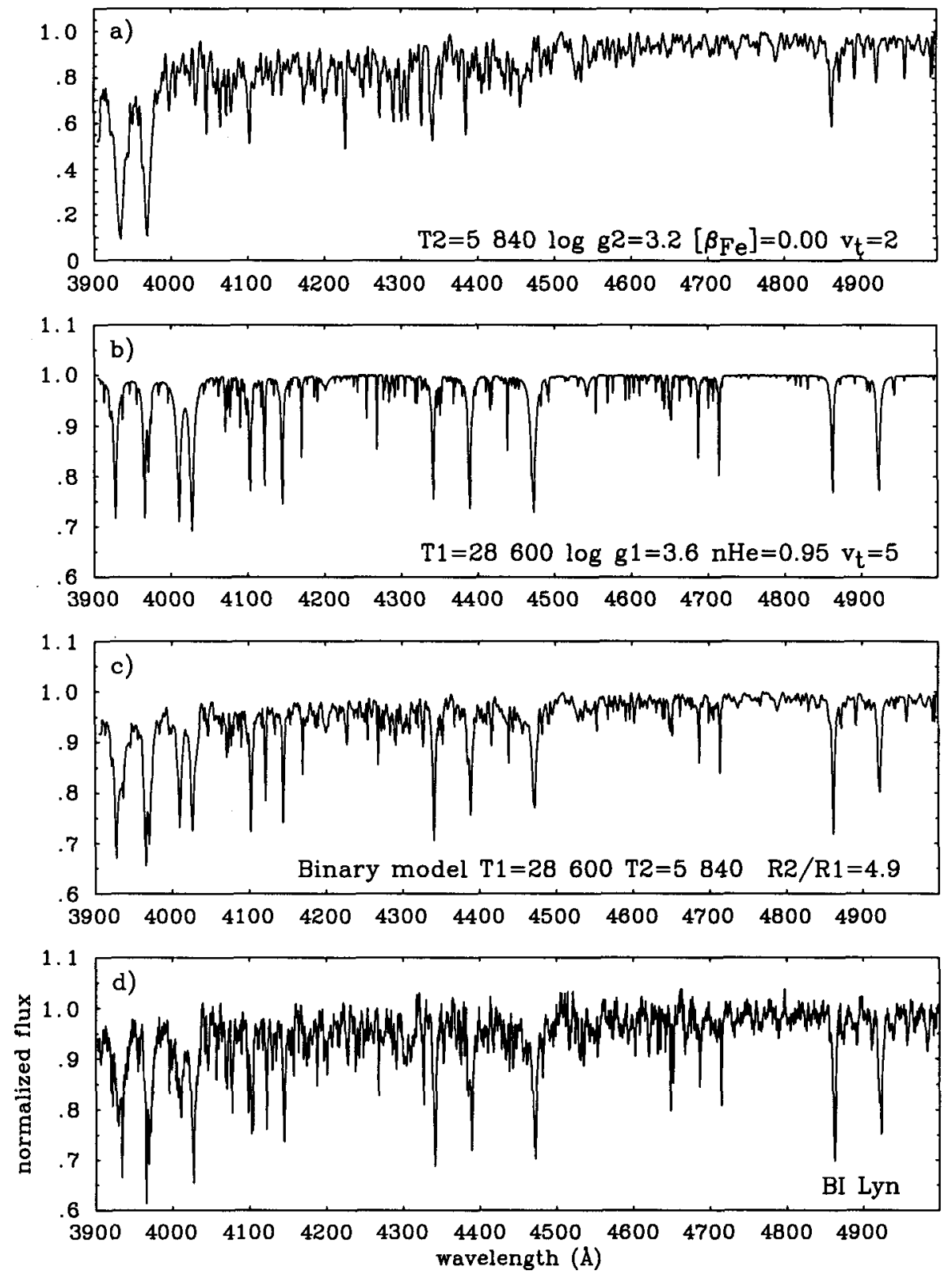

Figure 2. Normalized blue spectrum of BI Lyn (bottom: d) together with a best fit composite model spectrum (c) formed by adding models with a) $T_{\text {eff: } 2}=5840 \mathrm{~K}, \log g_{2}=3.2,[\mathrm{Fe} / \mathrm{H}]_{2}=0.00$ (top) and b) $T_{\text {eff: } 1}=28600 \mathrm{~K}, \log g_{1}=3.64, n_{\mathrm{He}: 1}=0.95$ assuming that the relative radii $R_{2} / R_{1}=4.9$. The model spectra have been velocity shifted and degraded to match the observed spectral resolution $(1 \AA)$. 
The analysis of the hot star based on the WHT blue spectrum yields a hydrogen abundance $\sim 1 \%$ by number, $T_{\text {eff }} \sim 28600 \mathrm{~K}$ and $\log g \sim 3.6$, making the primary a helium giant. Assuming a solar composition for the cool star, its surface gravity is estimated from the CaT lines to be $\log g \sim 3.2$ and hence also a giant. The two methods (FFIT and SFIT) give a radius ratio for the two stars $R_{2} / R_{1}$ between 7.4 and 4.9 . Simply estimating $M_{1}=0.5 \mathrm{M}_{\odot}$ enables all remaining system dimensions to be evaluated, including estimates for distance, luminosities and masses.

If it is assumed that the helium star mass is $\sim 0.5 \mathrm{M}_{\odot}$, and we adopt the radius ratio $R_{2} / R_{1} \sim 4.9 \pm 0.5$ from spectrophotometry, then we find radii of $1.9 \pm 0.3$ and $9.1 \pm 1.9 \mathrm{R}_{\odot}$ and luminosities $\left(\log L / L_{\odot}\right) 3.3 \pm 0.1$ and $1.9 \pm 0.1$ for the hot and cool stars, respectively. The cool star mass is more problematic; from the surface gravity we obtain $M_{2}=4.8 \pm 1.8 \mathrm{M}_{\odot}$, a lower limit would be $\approx 1.0 \pm 0.4 \mathrm{M}_{\odot}$.

\section{Conclusions}

Contrary to previous assumptions, BI Lyn does not contain a hot subdwarf or white dwarf, nor is it a nova-like variable. The hot star is a luminous hot hydrogen-deficient star with a probable mass around $0.5 \mathrm{M}_{\odot}$. Its luminosity suggests that it lies on a post-AGB evolution track. The cool star is a giant with a mass $>1 \mathrm{M}_{\odot}$. Of particular interest is the fact that it is rotating rapidly $\left(v \sin i=120 \pm 20 \mathrm{~km} \mathrm{~s}^{-1}\right)$.

The assumed mass is partially a result of the implied distance (>5 kpc) and consequent position in the Galaxy. At a relatively high Galactic latitude, a significantly larger total system mass is improbable from considerations of both the secondary spectral type and the unlikely location for a system with such a high mass.

It has been pointed out that the highly evolved star HD 128220 contains a Otype subdwarf and a rapidly-rotating G-type primary, probably spun up by mass exchange (Howarth \& Heber 1990). Both BI Lyn and HD 128220 thus consist of a hot post-AGB star and a more massive rapidly-rotating cool companion. Significantly, our estimates for the masses and luminosities of both components in BI Lyn agree well with the estimates given by Howarth \& Heber (1990) for HD 128220. The orbital period of HD 128220 is $871 \mathrm{~d}$. Similarly, we anticipate a long period and relatively small velocity amplitudes $\left(\$ 30 \mathrm{~km} \mathrm{~s}^{-1}\right)$ for BI Lyn.

The hydrogen deficiency of the hot star is probably the result of at least one common-envelope phase during which the outer envelope was entirely removed or partially transferred to the cool companion. The helium star must at one time have been substantially more massive than it is at present. Howarth \& Heber (1990) argue for an initial mass of $2 \mathrm{M}_{\odot}$ for the sdO star in HD 128220. After losing some $70 \%$ of the original primary mass and inverting the original mass ratio, the orbital separation would eventually increase towards the end of the mass-transfer phase until the stars were no longer in contact. Having lost its outer envelope, the primary can no longer sustain shell hydrogen burning and so enters the post-AGB phase. BI Lyn and HD 128220 are thus both long-period binaries which will ultimately comprise a white dwarf and a $\mathrm{G}$ star. The latter is 
effectively a blue straggler, having accreted a substantial fraction of the original primary during the mass transfer or common-envelope phase of evolution.

There remains one crucial difference between BI Lyn and HD 128220, namely the surface hydrogen abundance. HD 128220 has a helium to hydrogen ratio of 0.3 (Rauch 1993), compared with 20 for BI Lyn. If the two systems are genuinely comparable, then the mechanism that terminates mass transfer and the AGB phase must be able to produce a range of surface helium abundances. An important clue will be provided by measuring the orbital period and velocities for BI Lyn.

The previously-established $0.34 \mathrm{~d}$ light variations are likely to be due to pulsations in the helium star. By analogy with the hydrogen-deficient binary $v$ Sgr, previously established $\mathrm{H} \alpha$ P-Cygni profile variations may be due to the orbital motion of a supersonic jet between the components.

Further observations are required to determine the orbital period and mass ratio, to verify the pulsation hypothesis, to correlate the $\mathrm{H} \alpha$ behaviour with orbital phase and to establish the surface composition of both stars with greater precision.

Acknowledgments. This research is based on observations obtained with the Isaac Newton and William Herschel Telescopes, and on INES data from the IUE satellite. This research has made use of the SIMBAD database, operated at CDS, Strasbourg, France. CSJ is indebted to the Royal Society, London, and the International Astronomical Union for awarding travel grants to attend IAU Colloquium 187.

\section{References}

Aznar Cuadrado, R., \& Jeffery, C. S. 2001, A\&A, 368, 994

Aznar Cuadrado, R., \& Jeffery, C. S. 2002, A\&A, 385, 131

Downes, R., Webbink, R. F., \& Shara, M. M. 1997, PASP, 109345

Ferguson, D. H., Green, R. F., \& Liebert, J. 1984, ApJ, 287, 320

Howarth, I. D., \& Heber, U. 1990, PASP, 102, 912

Jeffery, C. S., \& Aznar Cuadrado, R. 2001, A\&A, 378, 936

Jeffery, C. S., \& Pollacco, D. 1998, MNRAS, 298, 179

Kuczawska, E., Mikolajewski, M., \& Kirejczyk K. 1993, IBVS, 3845

Lipunova, N. A., \& Shugarov, S. Yu. 1990, JAVSO, 19, 40

Lipunova, N. A., \& Shugarov, S. Yu. 1991, IBVS, 3580

Liu, W., \& Hu, J. Y. 2000, ApJS, 128, 387

Orosz, J. A., Wade, R. A., \& Harlow J. J. B. 1997, AJ, 114, 317

Rauch, T. 1993, A\&A, 276, 171

Wade, R. A., \& Potter, D. 1995, BAAS, 27, 877 